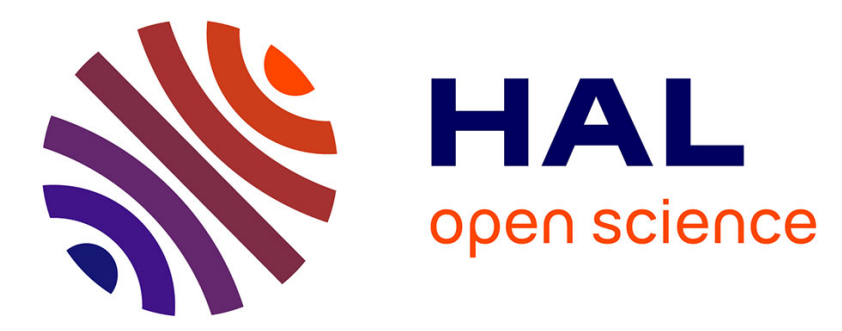

\title{
Monte Carlo investigation of how interfacial magnetic couplings affect blocking temperature distributions in exchange bias bilayers
}

\author{
G. Lhoutellier, D. Ledue, Renaud Patte, Vincent Baltz
}

\section{- To cite this version:}

G. Lhoutellier, D. Ledue, Renaud Patte, Vincent Baltz. Monte Carlo investigation of how interfacial magnetic couplings affect blocking temperature distributions in exchange bias bilayers. Journal of Applied Physics, 2016, 120, pp.193902. 10.1063/1.4967829 . hal-01683640

\author{
HAL Id: hal-01683640 \\ https://hal.science/hal-01683640
}

Submitted on 19 May 2019

HAL is a multi-disciplinary open access archive for the deposit and dissemination of scientific research documents, whether they are published or not. The documents may come from teaching and research institutions in France or abroad, or from public or private research centers.
L'archive ouverte pluridisciplinaire HAL, est destinée au dépôt et à la diffusion de documents scientifiques de niveau recherche, publiés ou non, émanant des établissements d'enseignement et de recherche français ou étrangers, des laboratoires publics ou privés. 


\title{
Monte Carlo investigation of how interfacial magnetic couplings affect blocking temperature distributions in exchange bias bilayers
}

\author{
G. Lhoutellier, ${ }^{1}$ D. Ledue, ${ }^{1, a)}$ R. Patte, ${ }^{1}$ and V. Baltz ${ }^{2,3,4, b)}$ \\ ${ }^{1}$ Normandie Univ., INSA Rouen, UNIROUEN, CNRS, GPM, 76000 Rouen, France \\ ${ }^{2}$ Univ. Grenoble Alpes, SPINTEC, F-38000 Grenoble, France \\ ${ }^{3}$ CNRS, SPINTEC, F-38000 Grenoble, France \\ ${ }^{4}$ CEA, INAC-SPINTEC, F-38000 Grenoble, France
}

(Received 13 July 2016; accepted 3 November 2016; published online 16 November 2016)

\begin{abstract}
Exchange bias in ferromagnetic (F)/antiferromagnetic (AF) bilayers is a function of both the bulk properties of the AF layer and the interfacial properties determining the effective interfacial couplings between the F and AF layers. The distinction between bulk and interface can be clearly revealed in blocking temperature distributions, where AF grain volume distribution results in a high-temperature peak while disordered interfacial magnetic phases produce a low-temperature contribution. However, the coupling conditions producing such bimodal blocking temperature distributions remain to be specified. In this article, we use a granular model which accounts for the disordered interfacial phases by considering small magnetic grains (SGs) with weaker anisotropy and coupling with the F grains at the F/AF interface. The SG are included in the AF material. The coupling conditions producing bimodal blocking temperature distributions were determined. Then, using Monte Carlo simulations, these conditions were validated and the effect of interfacial F-SG coupling on distributions was investigated. We next determined how the ratio between F-SG and F-AF couplings could be used to estimate the surface coverage of the disordered interfacial phases from experimental data. Published by AIP Publishing. [http://dx.doi.org/10.1063/1.4967829]
\end{abstract}

\section{INTRODUCTION}

Exchange bias refers to the magnetic exchange interactions between ferromagnetic $(\mathrm{F})$ and antiferromagnetic $(\mathrm{AF})$ materials in a F/AF bilayer. F/AF exchange biased bilayers are used in spintronic devices to set the reference direction determining the spin of conduction electrons. ${ }^{1,2}$ Exchange bias is determined by numerous parameters such as the bulk anisotropies, the bulk and interfacial exchange couplings, and the grain volumes in polycrystalline films. ${ }^{1,2}$ In the AF layer, two categories of magnetic phases are usually considered: bulk phases ${ }^{3-6}$ and interfacial phases with a certain level of surface coverage. ${ }^{7-13}$ These phases have low freezing temperatures due to magnetic frustration produced by defects at the F/AF interface, and are also known as disordered interfacial spins or low freezing temperature spins. These interfacial phases can significantly affect the exchange bias properties. At working temperatures for devices, they no longer contribute to the exchange field. ${ }^{14}$

Experimentally, the magnetic phases in the AF layer are accessible through several techniques, for example, they can be deduced from thermoremanent studies down to low temperatures. ${ }^{10}$ Although this method is appropriate for isolated $\mathrm{AF}$ materials, it does not work for F/AF bilayers because the signal from the $\mathrm{F}$ layer screens that of the disordered phases. Another technique, $\mathrm{x}$-ray magnetic dichroism, can measure uncompensated AF spins in magnetic phases. ${ }^{9}$ However, it requires large-scale facilities, and $\mathrm{x}$-ray absorption by the

\footnotetext{
a)denis.ledue@univ-rouen.fr

b)vincent.baltz@cea.fr
}

top layers in a stack may create difficulties. An alternative is to measure blocking temperature distributions to identify magnetic phases in the AF layer. ${ }^{5,8}$ This technique presents no particular technical difficulties, and can be performed, for example, using the stepwise procedure described by Soeya et $a l .{ }^{5}$ In this procedure, the sample is first field-cooled from above the maximum blocking temperature down to $4 \mathrm{~K}$ under a positive field. This cooling causes the magnetic phases of the AF layer in contact with the F layer to align in the positive direction. Then, the sample's temperature is raised to an intermediate annealing temperature $T_{\mathrm{a}}$, and the sample is subsequently field-cooled under a negative field down to $4 \mathrm{~K}$. This cooling step results in a negative reorientation of the magnetic phases of the AF layer in contact with the F layer, when the blocking temperature is lower than $T_{\mathrm{a}}$. Finally, a hysteresis loop is measured at $4 \mathrm{~K}$. These steps are repeated for a range of $T_{\mathrm{a}}$ and the variation in the exchange field, $H_{\mathrm{E}}$, with $T_{\mathrm{a}}$ and its derivative $d H_{\mathrm{E}} / d T_{\mathrm{a}}$ are recorded. Typical experimental results for $\operatorname{Co}(3 \mathrm{~nm}) / \operatorname{IrMn}(7 \mathrm{~nm})$ bilayers ${ }^{15}$ have been reproduced in Fig. 1. Since the increase in the exchange field with $T_{\mathrm{a}}$ is related to the negative repolarization of the magnetic phases of the AF layer in contact with the $\mathrm{F}$ layer, the derivative $d H_{\mathrm{E}} / d T_{\mathrm{a}}$, is linked to the blocking temperature distributions of these magnetic phases. $^{5,8}$ The volume distribution of grains in the AF bulk phases in contact with the $\mathrm{F}$ layer gives rise to a hightemperature peak; a low-temperature contribution is also observed, which is attributed to the interfacial phases. The relative contribution from the interfacial phases can be extracted from the relative weights of the high- and lowtemperature contributions. ${ }^{14}$ However, the impact of the 

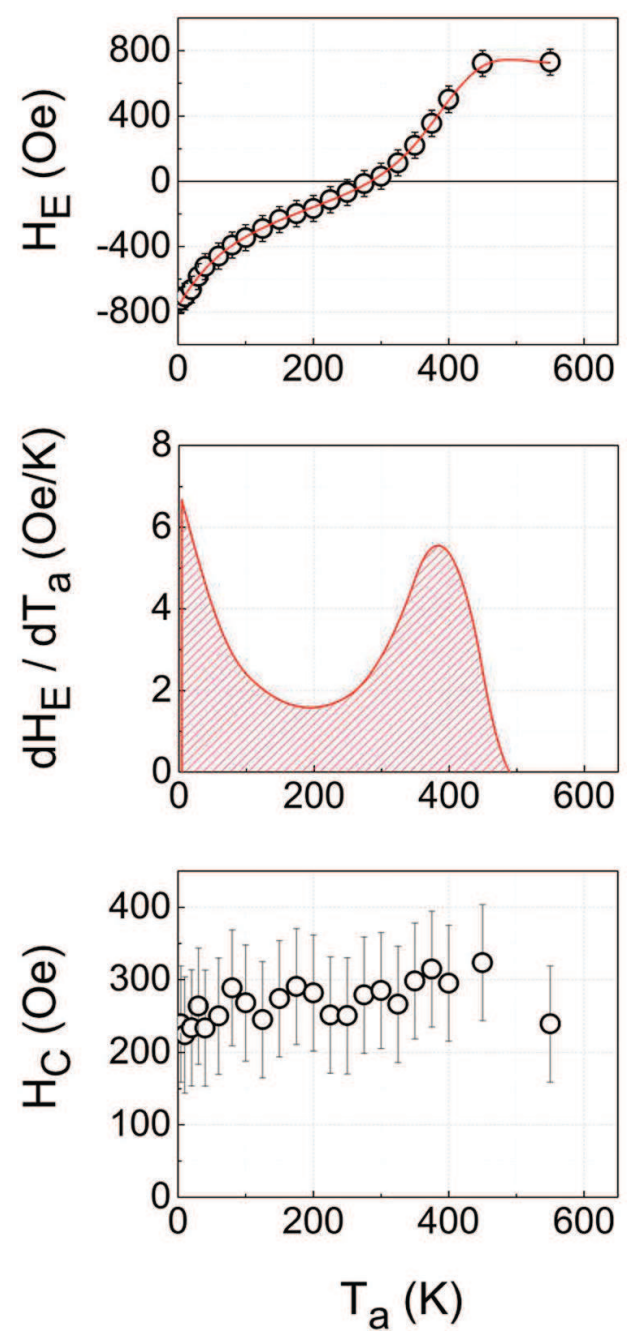

FIG. 1. Experimental dependences of $H_{\mathrm{E}}$ (top, the full lines result from interpolation of the data), the derivative $d H_{\mathrm{E}} / d T_{\mathrm{a}}$ (middle, from the interpolation) and $H_{\mathrm{C}}$ (bottom) on $T_{\mathrm{a}}$ for $\operatorname{Co}(3 \mathrm{~nm}) / \operatorname{IrMn}(7 \mathrm{~nm})$ bilayers (from Ref. 15).

interfacial couplings on each contribution remains to be determined.

From a theoretical point of view, it is not easy to account for such double-peaked $d H_{\mathrm{E}} / d T_{\mathrm{a}}$. For example, attempts were made to use a single effective F/AF coupling which slowly decreases as the temperature increases. ${ }^{3}$ Unfortunately, this model produced a single peak. Recently, a Monte Carlo investigation based on a granular model ${ }^{16}$ validated the hypothesis that less stable magnetic regions in the AF layer, located at the F/AF interface, could be responsible for the low-temperature peak of $d H_{\mathrm{E}} / d T_{\mathrm{a}}$ and thus, the bimodal distribution of blocking temperature, experimentally observed.

In the present study, using a granular model, we, first, analytically determined the interfacial coupling conditions required to obtain a double-peaked derivative $d H_{\mathrm{E}} / d T_{\mathrm{a}}$. These predictions were subsequently validated using Monte Carlo simulations which are very suitable for simulating the procedure described above. ${ }^{5}$ In addition, we discuss the relationship between the derivative $d H_{\mathrm{E}} / d T_{\mathrm{a}}$ and the blocking temperature distribution. We also indicate how our results may be useful in refining how the relative amount of interfacial magnetic phases can be determined from experimental data.

\section{MODEL AND SIMULATION}

Our model bilayer (Fig. 2) consisted of one F layer and one AF layer with the same microstructure since we assumed columnar growth. For each layer, the granular microstructure was generated by Voronoi tessellation ${ }^{17}$ in two-dimensions and the resulting volume distribution was log-normal. The average volume is $(56.25 \times t) \mathrm{nm}^{3}$ where $t$ is the layer thickness. The standard deviation $\sigma$ is about 0.43 and the median grain size is $(51.28 \times t) \mathrm{nm}^{3}$. Smaller grains (SGs) with reduced anisotropy compared to the bulk AF grains were randomly spread over the F/AF interface within the AF (Fig. 2). The surface coverage of these $S G$ was expressed as a percentage of the total surface, $x_{\mathrm{SG}}$. The $\mathrm{SG}$ account for the magnetic phases with low freezing temperatures due to magnetic frustration produced by defects at the F/AF interface (e.g., interlayer diffusion and stacking faults). Due to that, the SG are not compositionally similar to the AF but show deteriorated magnetic properties. Since the dimensions of the grains are in the nanometer scale, every grain was assumed to support a single magnetic domain that reverses by uniform rotation. Thus, a unit vector, $\boldsymbol{\sigma}_{i}$, represents the magnetization orientation of each F grain, each SG and the interfacial uncompensated magnetization orientation of each bulk AF grain. Across the interface, each $\mathrm{F}$ grain was coupled with one AF grain $\left(J_{\mathrm{F}-\mathrm{AF}}\right)$, or with one $\mathrm{SG}\left(J_{\mathrm{F}-\mathrm{SG}}\right)$. In addition, each $\mathrm{SG}$ was coupled with one AF grain below $\left(J_{\mathrm{AF}-\mathrm{SG}}\right)$. In agreement with the literature, the $\mathrm{AF}$ grains were not linked to each other, ${ }^{6,18,19}$ and nor were the SG. In contrast, the $\mathrm{F}$ grains were coupled to each other $\left(J_{\mathrm{F}}\right)$ (Fig. 2). Uniaxial anisotropy along a common easy axis ( $y$-axis) in the plane of the layer was considered for all grains, and a linear thermal dependence of the anisotropy constants per unit volume $K_{\mathrm{i}}$, was implemented. ${ }^{20}$ Thus, the total energy of the system can be expressed as

$$
E=-\sum_{\langle i, j\rangle} J_{i j} \boldsymbol{\sigma}_{i} \cdot \boldsymbol{\sigma}_{j}-\sum_{i} K_{i} V_{i}\left(\boldsymbol{\sigma}_{i} \cdot \mathbf{e}_{y}\right)^{2}-\mu_{0} \mathbf{H} \cdot \sum_{i \in F, S G} \mathbf{m}_{i}
$$

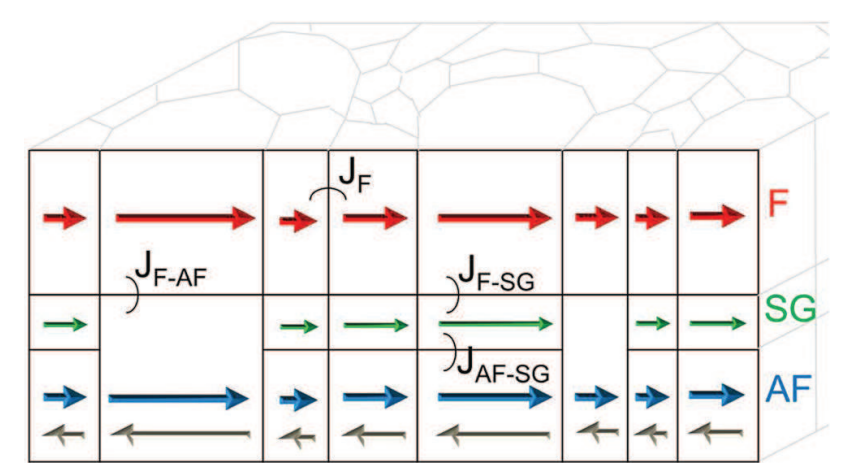

FIG. 2. Schematic representation of the F/AF bilayer. The model considers $\mathrm{F}$ and $\mathrm{AF}$ grains as well as smaller grains (SG) present at the F/AF interface. 
where $V_{\mathrm{i}}$ is the grain volume, $\mathbf{m}_{\mathrm{i}}$ is the magnetic moment of the $\mathrm{F}$ grain or $\mathrm{SG}$, and $\mathbf{H}$ is the field applied along the $y$-axis.

Next, the protocol described by Soeya et al..$^{5}$ was simulated using the Monte Carlo method. ${ }^{21,22}$ At each temperature (during the field-cooling) or at each value of the applied field (during the hysteresis loop), $n$ Monte Carlo steps are performed, that is, each grain is visited $n$ times (typically, $n$ is several thousand). At each visit, a magnetization reversal trial with an acceptance rate equal to $\exp \left(-\Delta E /\left(k_{B} T\right)\right)$, where $\Delta E$ is the energy barrier to overcome during reversal, is made. ${ }^{16,23}$ Readers should remember that these energy barriers mainly depend on the anisotropy and also on the local field acting on the grain considered. In the Monte Carlo method, the time unit is one Monte Carlo step. Unfortunately, the physical time $\delta t$ corresponding to one Monte Carlo step is, in general, unknown. However, in our particular case, it has been found that $\delta t \approx 2 \tau_{0}{ }^{24}$ where $\tau_{0}$ is the prefactor in the expression of the relaxation time $\tau=\tau_{0} e^{\frac{K V}{K_{B} T}}$. Consequently, the physical time corresponding to $n$ Monte Carlo steps is $n \delta t \approx 2 n \tau_{0}$, which corresponds to the measuring time $t_{\mathrm{m}}$.

To compare our results with available experimental results, ${ }^{8,14,15}$ we used 5-nm-thick F and AF layers. Assuming that the interfacial magnetic phases extended over 3-4 atomic planes, we set the thickness of the $\mathrm{SG}, t_{\mathrm{SG}}$, to $1 \mathrm{~nm},{ }^{25,26}$ thus, reducing the thickness of the AF grains located below them to $4 \mathrm{~nm}$ (see Fig. 2). Since the study was based on $\mathrm{Co} / \mathrm{IrMn}$ bilayers, the transition temperatures used in the expression of the anisotropy constants ${ }^{20}$ were $T_{\mathrm{C}}=1360 \mathrm{~K}$ for the $\mathrm{F}$ grains $(\mathrm{Co})$ and $T_{\mathrm{N}}=690 \mathrm{~K}$ for the AF grains (IrMn). ${ }^{1}$ The $0 \mathrm{~K}$ anisotropy constant for the $\mathrm{AF}$ grains was estimated to be $K_{A F}^{0}=9.8 \times 10^{5} \mathrm{~J} \mathrm{~m}^{-3}{ }^{27}$ We then took $K_{F}^{0}=7.2 \times 10^{4} \mathrm{~J} \mathrm{~m}^{-3}$ so that the intrinsic blocking temperature would be $140 \mathrm{~K}$ for a $\mathrm{F}$ grain of volume equal to the average volume, $\bar{V}_{F}=(7.5 \times 7.5 \times 5) \mathrm{nm}^{3}$. The effective anisotropy of the SG was assumed to be weaker than that of the AF grains; we, therefore, arbitrarily set $K_{S G}^{0}$ to $0.3 K_{A F}^{0}$. As a basis for further discussion, we calculated the intrinsic blocking temperature distribution for the AF grains and SG, that is, the blocking temperatures in the absence of coupling and applied field. The intrinsic blocking temperature $T_{\mathrm{B} \text {,int }}$, of a grain is defined by $\tau\left(T_{\mathrm{B}, \text { int }}\right)=t_{\mathrm{m}}$, where $\tau=\tau_{0} e^{\frac{K V}{K_{B} T}}$. The intrinsic blocking temperatures of the AF grains and of the SG can thus be written as

$$
T_{B, \text { int }}=\frac{K V}{k_{B} \ln \left(t_{m} / \tau_{0}\right)},
$$

where $K=K_{\mathrm{AF}}$ or $K_{\mathrm{SG}}$. Since, in our simulations, $t_{\mathrm{m}} \approx 2 n$ $\tau_{0}$, we could calculate the intrinsic blocking temperature distribution of the grains from their volume distributions. These distributions for $n=5.5 \times 10^{3}$ are plotted in Fig. 3. The intrinsic blocking temperatures of an $\mathrm{AF}$ grain of volume equal to the average volume $\bar{V}_{A F}=(7.5 \times 7.5 \times 5) \mathrm{nm}^{3}$, and a SG grain of volume equal to its average volume $\bar{V}_{S G}=(7.5 \times 7.5 \times 1) \mathrm{nm}^{3}$, will be $T_{B \text {,int }}^{A F}=521.6 \mathrm{~K}$ and $T_{B \text {,int }}^{S G}=108.4 \mathrm{~K}$, respectively. Below, we will show that even though the intrinsic blocking temperature distribution is bimodal, it is not guaranteed that $d H_{\mathrm{E}} / d T_{\mathrm{a}}$, obtained from

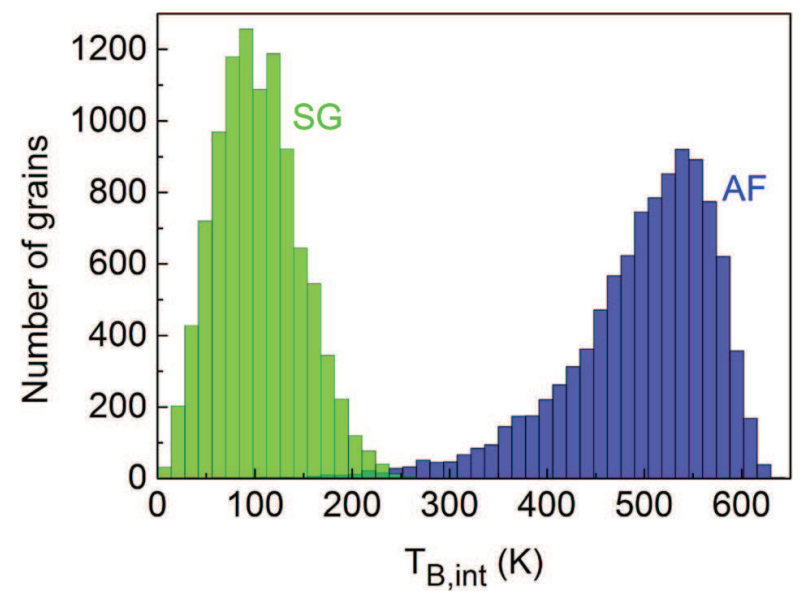

FIG. 3. Intrinsic blocking temperature distributions for the SG and AF grains calculated from the volume distribution simulated for $N=10^{4}$ grains.

Ref. 5 procedure, will have a double-peak, since this characteristic depends on the degree of coupling between grains.

The effective interfacial coupling per unit area $j_{\mathrm{F}-\mathrm{AF}}$, is simply related to the exchange field in the case of an ideal $\mathrm{F} /$ AF interface, that is, without SG. Here, $j_{\mathrm{F}-\mathrm{AF}}$ was chosen so that the value of $H_{\mathrm{E}}$ at $0 \mathrm{~K}$, when all $\mathrm{AF}$ grains are polarized in the same direction, would be equal to $700 \mathrm{Oe}$, in agreement with previous experimental studies ${ }^{15}$ (Fig. 1). We, therefore, took $j_{\mathrm{F}-\mathrm{AF}}=5 \times 10^{-22} \mathrm{~J} \mathrm{~nm}^{-2}$. The actual couplings per unit area, $j_{\mathrm{F}-\mathrm{SG}}$ and $j_{\mathrm{AF}-\mathrm{SG}}$, are unknown. However, $j_{\mathrm{F}-\mathrm{SG}}$ is assumed to be smaller than $j_{\mathrm{F}-\mathrm{AF}}$. In addition, $j_{\mathrm{F}-\mathrm{SG}}$ and $j_{\mathrm{AF}-\mathrm{SG}}$ must be chosen such that: (i) the SG should be partially repolarized toward the negative direction when field-cooling is applied (the fraction of SG that will be repolarized depends on $T_{\mathrm{a}}$ and on the field applied $\mathbf{H}_{F C}$ ) and (ii) the coercive field should not vary with $T_{\mathrm{a}}$, that is, with the fraction of repolarized entities in contact with the $F$ layer. This second condition is required for the model to be consistent with the experimental data ${ }^{5,8,15}$ (Fig. 1). Condition (i) is fulfilled if and only if the local field (including the applied field) acting on a SG is negative and smaller in absolute value than the anisotropy field $H_{a}^{S G}=\frac{2 K_{S G}}{\mu_{0} M_{S G}}\left(M_{\mathrm{SG}}\right.$ is the magnetization of a SG, $M_{\mathrm{SG}}=164 \mathrm{kA} \mathrm{m}^{-1}=M_{\mathrm{AF}} / 8$ to take into account magnetic disorder such as interlayer diffusion and stacking faults). Since the local field acting on a SG is the sum of the field due to the $\mathrm{F}$ grain in contact $\mathbf{H}_{F-S G}=$ $\frac{J_{F-S G}}{\mu_{0} M_{S G} V_{i}} \boldsymbol{\sigma}_{F}$ (with $J_{\mathrm{F}-\mathrm{SG}}>0$ and $\boldsymbol{\sigma}_{F}=-\mathbf{e}_{y}$ ), the field due to the AF grain in contact $\mathbf{H}_{A F-S G}=\frac{J_{A F-S G}}{\mu_{0} M_{S G} V_{i}} \boldsymbol{\sigma}_{A F}$ (with $J_{\mathrm{AF}-\mathrm{SG}}>0$ and $\boldsymbol{\sigma}_{A F}=\mathbf{e}_{y}$ ), and $\mathbf{H}_{F C}$ (Fig. 4(a)), this condition can be written as

$$
-H_{a}^{S G}<-\frac{J_{F-S G}}{\mu_{0} M_{S G} V_{i}}+\frac{J_{A F-S G}}{\mu_{0} M_{S G} V_{i}}-\left|H_{F C}\right|<0,
$$

with $V_{\mathrm{i}}=S_{\mathrm{i}} t_{\mathrm{SG}}\left(S_{\mathrm{i}}\right.$ is the surface of the $\left.\mathrm{SG}\right)$. The condition can be rewritten as

$$
0<j_{\mathrm{F}-\mathrm{SG}}-j_{\mathrm{AF}-\mathrm{SG}}+\mu_{0} M_{\mathrm{SG}} t_{\mathrm{SG}}\left|H_{F C}\right|<2 K_{\mathrm{SG}}(T) t_{\mathrm{SG}} .
$$

Condition (ii) requires that the negatively repolarized SG should not switch magnetization after the $\mathrm{F}$ grains have 


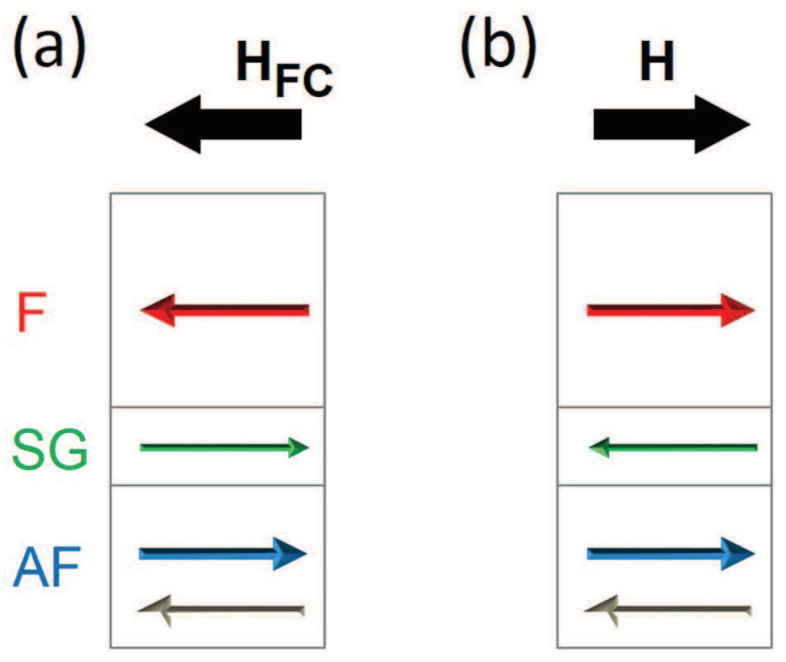

FIG. 4. Schematic representation of the F/AF bilayer during (a) field cooling before negative reorientation of the SG and (b) the hysteresis loop at $4 \mathrm{~K}$ after reversal of the magnetization from negative to positive direction in the F layer.

reversed their magnetization from negative to positive orientation during the hysteresis loops (right hand side of the hysteresis loop). Thus, the local field (including the applied field) acting on a SG after the F grains have reversed (Fig. 4(b)) should be smaller than the anisotropy field $H_{a}^{S G}$. This condition can be written as

$$
0<\frac{J_{F-S G}}{\mu_{0} M_{S G} V_{i}}+\frac{J_{A F-S G}}{\mu_{0} M_{S G} V_{i}}+H<H_{a}^{S G}
$$

where $H>0$ is the field applied during the hysteresis loop, and thus

$$
0<j_{\mathrm{F}-\mathrm{SG}}+j_{\mathrm{AF}-\mathrm{SG}}+\mu_{0} M_{\mathrm{SG}} t_{\mathrm{SG}} H<2 K_{\mathrm{SG}}(T) t_{\mathrm{SG}} .
$$

It should be noted that this inequality is a necessary condition, but the coercive field can vary if the $\mathrm{F}$ layer fails to reverse by uniform rotation. Inequalities (1) and (2) are summarized in Fig. 5. According to this diagram, only the conditions represented in the green area (lower left corner) will produce double-peaked $d H_{\mathrm{E}} / d T_{\mathrm{a}}$ with a constant coercive field as $T_{\mathrm{a}}$ varies (assuming uniform rotation of the $\mathrm{F}$ layer). We remark that increasing the anisotropy constant of the SG is equivalent to decreasing $j_{\mathrm{F}-\mathrm{SG}}$ and $j_{\mathrm{AF}-\mathrm{SG}}$ and to shifting upward the blue long-dashed line and to shifting downward the black full and the red short-dashed lines in Fig. 5.

\section{RESULTS AND DISCUSSION}

To check whether in the conditions indicated, doublepeaked or single-peaked $d H_{\mathrm{E}} / d T_{\mathrm{a}}$ will be produced, we considered three pairs of (reduced) couplings $\left(j_{\mathrm{F}-\mathrm{SG}} /\left(K_{S G}^{0} t_{S G}\right)\right.$, $\left.j_{\mathrm{AF}-\mathrm{SG}} /\left(K_{S G}^{0} t_{S G}\right)\right)$ : $(1.8,0.1)$ (blue area in Fig. 5); $(0.2,1.2)$ (orange area in Fig. 5); and $(0.2,0.1)$ (green area in Fig. 5).

The simulated variation of $H_{\mathrm{E}}$ vs. $T_{\mathrm{a}}$ was obtained from the simulated hysteresis loops at $4 \mathrm{~K}$ (not shown here). Then, we numerically calculated the derivative of its interpolation. The variation in $H_{\mathrm{E}}$ and its derivative were plotted against $T_{\mathrm{a}}$ for $j_{\mathrm{F}-\mathrm{SG}} /\left(K_{S G}^{0} t_{S G}\right)=1.8$ and $j_{\mathrm{AF}-\mathrm{SG}} /\left(K_{S G}^{0} t_{S G}\right)=0.1$, and $j_{\mathrm{F}-\mathrm{SG}} /$

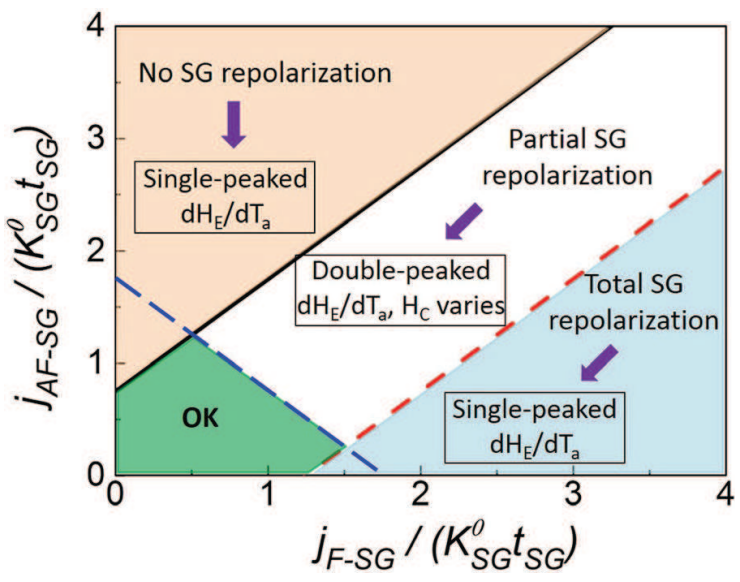

FIG. 5. $d H_{\mathrm{E}} / d T_{\mathrm{a}}$ as a function of the reduced variables $j_{\mathrm{F}-\mathrm{SG}} /\left(K_{S G}^{0} t_{S G}\right)$ and $j_{\mathrm{AF}-\mathrm{SG}} /\left(K_{S G}^{0} t_{S G}\right)$ (the black full line corresponds to a local field acting on a $\mathrm{SG}$ equal to 0 , the increasing red short-dashed line to a negative local field acting on a SG whose absolute value is equal to the anisotropy field $H_{a}^{S G}$, after negative repolarization of the $\mathrm{F}$ layer during field cooling, and the decreasing blue long-dashed line to a local field acting on a SG equal to the anisotropy field $H_{a}^{S G}$, after reversal of the magnetization in the F layer during the hysteresis loop). The green zone (lower left corner) represents the conditions in which $d H_{\mathrm{E}} / d T_{\mathrm{a}}$ will display a double peak.

$\left(K_{S G}^{0} t_{S G}\right)=0.2$ and $j_{\mathrm{AF}-\mathrm{SG}} /\left(K_{S G}^{0} t_{S G}\right)=1.2$ (Fig. 6, left and middle columns, respectively). In these figures, as expected, there is no peak at low-temperature, even though SG are present in large amounts $\left(x_{\mathrm{SG}}=75 \%\right)$ at the F/AF interface. However, the reasons for the absence of a low-temperature peak are not the same in the two different cases. Thus, in Fig. 6 (left column), there is no peak at low-temperature because, due the strong $j_{\mathrm{F}-\mathrm{SG}}$ coupling, all SG are repolarized by the $\mathrm{F}$ grains during field-cooling even for $T_{\mathrm{a}}=4 \mathrm{~K}$. Therefore, even if there is no visible peak at low-temperature, the exchange field is affected by the SG at room temperature. The absence of a low-temperature peak in Fig. 6 (middle column) is because the SG cannot be repolarized while the AF grains are not repolarized due to the strong $j_{\mathrm{AF}-\mathrm{SG}}$ coupling in this case. In these conditions, the SG start to be repolarized only from $T_{\mathrm{a}}=270 \mathrm{~K}$. The contribution of the $\mathrm{SG}$ to the derivative $d H_{\mathrm{E}} /$ $d T_{\mathrm{a}}$ is masked by the contribution of the AF grains, and the exchange field at room temperature is not affected by the SG.

Thus, a double-peaked $d H_{\mathrm{E}} / d T_{\mathrm{a}}$ is expected only for the set $j_{\mathrm{F}-\mathrm{SG}} /\left(K_{S G}^{0} t_{S G}\right)=0.2$ and $j_{\mathrm{AF}-\mathrm{SG}} /\left(K_{S G}^{0} t_{S G}\right)=0.1$. The variation of $H_{\mathrm{E}}$ and its derivative $d H_{\mathrm{E}} / d T_{\mathrm{a}}$ in these conditions is shown in Fig. 6 (right column). In agreement with the predictions from our analytical calculations, two contributions are observed. In addition, the locations of the two peaks are in good agreement with the intrinsic blocking temperature distributions shown in Fig. 3, thus, confirming the validity of the procedure. However, a slight shift of the low-temperature peak toward lower than expected temperatures is observed due to the $J_{\mathrm{F}-\mathrm{SG}}$ coupling which lowers the energy barrier for negative repolarization of the SG. We will discuss the relative heights of the two peaks below. In addition, the coercive field is independent of $T_{\mathrm{a}}$ as in previous experimental work ${ }^{5,8,15}$ (Fig. 1).

The fraction of SG $\left(x_{\mathrm{SG}}\right)$ at the interface needs to be extracted from the area of the low-temperature peak of $d H_{\mathrm{E}} /$ $d T_{\mathrm{a}}$ which is the quantity measured experimentally. To see 

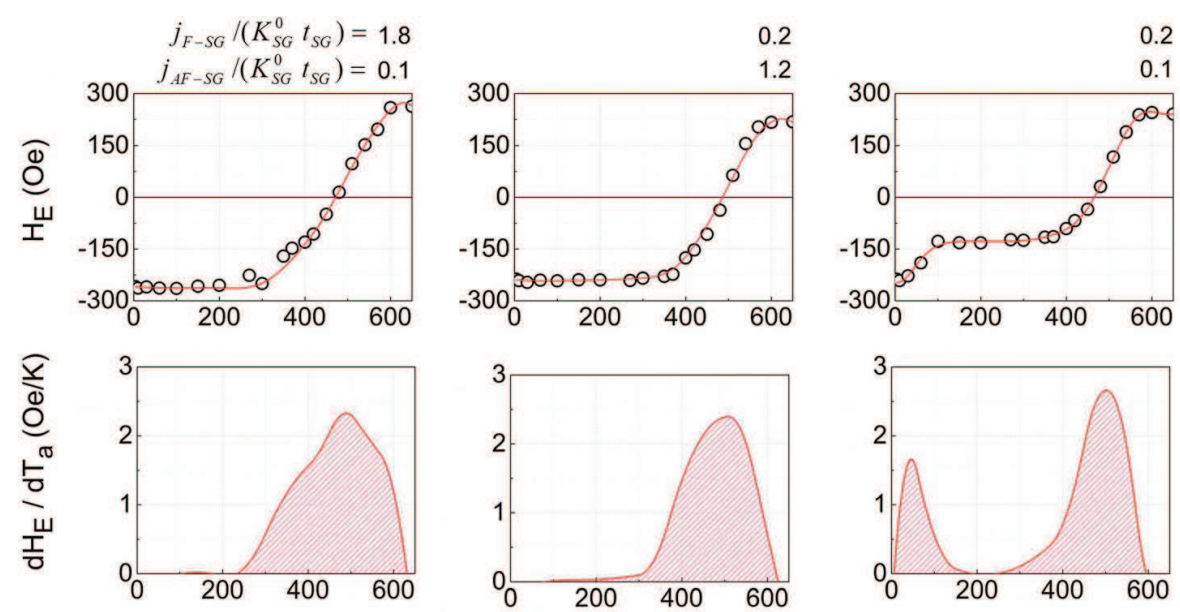

FIG. 6. Variation of $H_{\mathrm{E}}$ vs. $T_{\mathrm{a}}$, the full line is an interpolation of the data (top row), derivative $d H_{\mathrm{E}} / d T_{\mathrm{a}}$ vs. $T_{\mathrm{a}}$ (middle row, from the data interpolation) and variation of $H_{\mathrm{C}}$ vs. $T_{\mathrm{a}}$ (bottom row) for three pairs of (reduced) couplings $\quad\left(j_{\mathrm{F}-\mathrm{SG}} /\left(K_{S G}^{0} t_{S G}\right), \quad j_{\mathrm{AF}-\mathrm{SG}} /\right.$ $\left(K_{S G}^{0} t_{S G}\right)$ ) and $x_{\mathrm{SG}}=75 \%$.
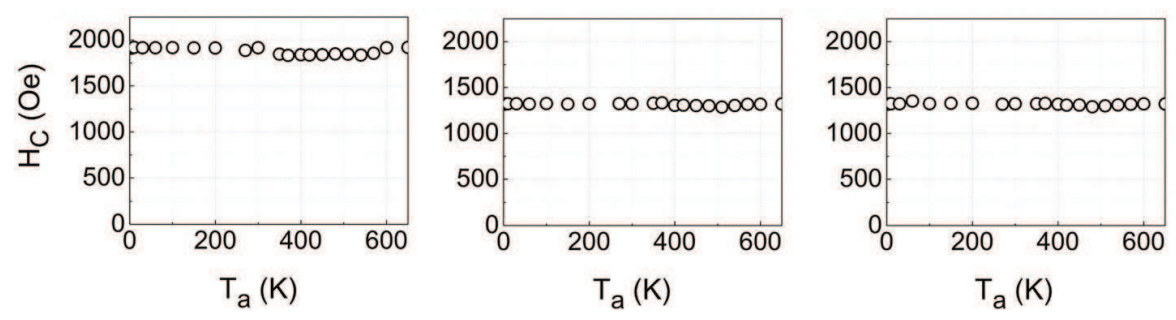

how this can be done, we investigated the effect of the $j_{\mathrm{F}-\mathrm{SG}}$ coupling $\left(j_{\mathrm{F}-\mathrm{SG}} /\left(K_{S G}^{0} t_{S G}\right)=0.1,0.2\right.$, and 0.4 , that is, $j_{\mathrm{F}-\mathrm{SG}} /$ $j_{\mathrm{F}-\mathrm{AF}}=0.05,0.1$, and 0.2$)$ on the derivative $d H_{\mathrm{E}} / d T_{\mathrm{a}}$ for $x_{\mathrm{SG}}=75 \%$ and $j_{\mathrm{AF}-\mathrm{SG}} /\left(K_{S G}^{0} t_{S G}\right)=0.1$ (Fig. 7). From this representation, it is clear that as $j_{\mathrm{F}-\mathrm{SG}}$ increases, the low- temperature peak of the derivative related to the SG grows, whereas the high-temperature peak (of the derivative) due to the $\mathrm{AF}$ grains remains constant. If we define the quantity $\Delta^{*}=S_{\mathrm{L}} /\left(S_{\mathrm{H}}+S_{\mathrm{L}}\right)$, where $S_{\mathrm{L}}$ and $S_{\mathrm{H}}$ are the surfaces of the low- and high-temperature peaks, respectively (Fig. 7), we obtain $\Delta^{*}=13 \%, 22 \%$, and $35 \%$ for $j_{\mathrm{F}-\mathrm{SG}} / j_{\mathrm{F}-\mathrm{AF}}=0.05,0.1$, and 0.2 , respectively. It should be noted that, as $j_{\mathrm{F}-\mathrm{SG}}$ increases, for the blocking temperature distribution, only a small shift toward low temperatures and no growth of the low-temperature peak is expected; the high-temperature peak should be unaltered because the blocking temperatures

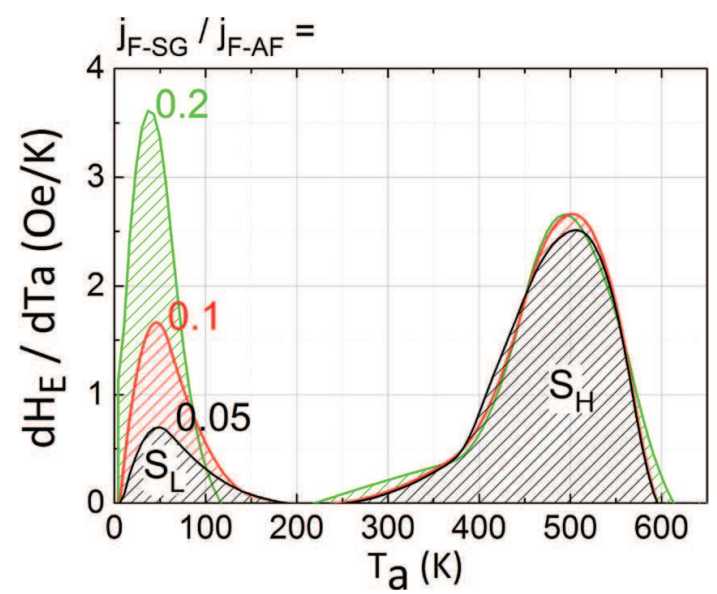

FIG. 7. Derivative $d H_{\mathrm{E}} / d T_{\mathrm{a}}$ calculated from $H_{\mathrm{E}}$ vs. $T_{\mathrm{a}}$ for various intensities of F-SG coupling, $j_{\mathrm{F}-\mathrm{SG}} / j_{\mathrm{F}-\mathrm{AF}}=0.05,0.1$, and 0.2 , with $x_{\mathrm{SG}}=75 \%\left(S_{\mathrm{L}}\right.$ and $S_{\mathrm{H}}$ correspond to the surfaces of the low- and high-temperature peaks).

of the AF grains are not affected by $j_{\mathrm{F}-\mathrm{SG}}$. The results presented above clearly indicate that the derivative $d H_{\mathrm{E}} / d T_{\mathrm{a}}$ is not proportional to the blocking temperature distribution.

To define how $x_{\mathrm{SG}}$, the quantity of interest, is related to the experimentally measured $\Delta^{*}$, we calculated the expression of $d H_{\mathrm{E}} / d T_{\mathrm{a}}$ assuming a uniform rotation of the $\mathrm{F}$ layer (see Appendix A), and found

$$
\begin{aligned}
\frac{d H_{E}\left(T_{a}\right)}{d T_{a}}= & \frac{2}{\mu_{0} M_{F} t_{F}}\left[x_{S G} j_{F-S G} P_{S G}\left(T_{a}\right)\right. \\
& \left.+\left(1-x_{S G}\right) j_{F-A F} P_{A F}\left(T_{a}\right)\right]
\end{aligned}
$$

where $M_{\mathrm{F}}$ is the magnetization of each $\mathrm{F}$ grain, $t_{\mathrm{F}}$ is the thickness of the F layer, and $P_{\mathrm{SG}}$ and $P_{\mathrm{AF}}$ are the normalized blocking temperature distributions of the SG and AF grains, respectively $\left(\int_{0}^{+\infty} P_{S G}(T) d T=\int_{0}^{+\infty} P_{A F}(T) d T=1\right)$. The surface fraction of the low-temperature contribution can readily be obtained from the above expression of $d H_{\mathrm{E}} / d T_{\mathrm{a}}$ (see Appendix B), thus

$$
\Delta^{*}=\left[x_{\mathrm{SG}} j_{\mathrm{F}-\mathrm{SG}} / j_{\mathrm{F}-\mathrm{AF}}\right] /\left[1-x_{\mathrm{SG}}+x_{\mathrm{SG}} j_{\mathrm{F}-\mathrm{SG}} / j_{\mathrm{F}-\mathrm{AF}}\right] .
$$

The variation in $\Delta^{*}$ and its relationship with $x_{\mathrm{SG}}$ for various values of $j_{\mathrm{F}-\mathrm{SG}} / j_{\mathrm{F}-\mathrm{AF}}$ is illustrated in Fig. 8 . From this representation, it is clear that $\Delta^{*}$ is lower than $x_{\mathrm{SG}}$ assuming $j_{\mathrm{F}-\mathrm{SG}}<j_{\mathrm{F}-\mathrm{AF}}$, and this difference increases as $j_{\mathrm{F}-\mathrm{SG}} / j_{\mathrm{F}-\mathrm{AF}}$ decreases in agreement with the simulated $\Delta^{*}$ values for $j_{\mathrm{F}-\mathrm{SG}} / j_{\mathrm{F}-\mathrm{AF}}=0.05,0.1$, and $0.2\left(x_{\mathrm{SG}}=75 \%\right)$ (Fig. 8). Note that $\Delta^{*}=x_{\mathrm{SG}}$ only if $j_{\mathrm{F}-\mathrm{SG}}=j_{\mathrm{F}-\mathrm{AF}}$. This was to be expected because in that case $d H_{\mathrm{E}} / d T_{\mathrm{a}}$ is proportional to the blocking temperature distribution.

\section{CONCLUSION}

In the framework of a granular model, and using Monte Carlo simulations, we specified some conditions on the 


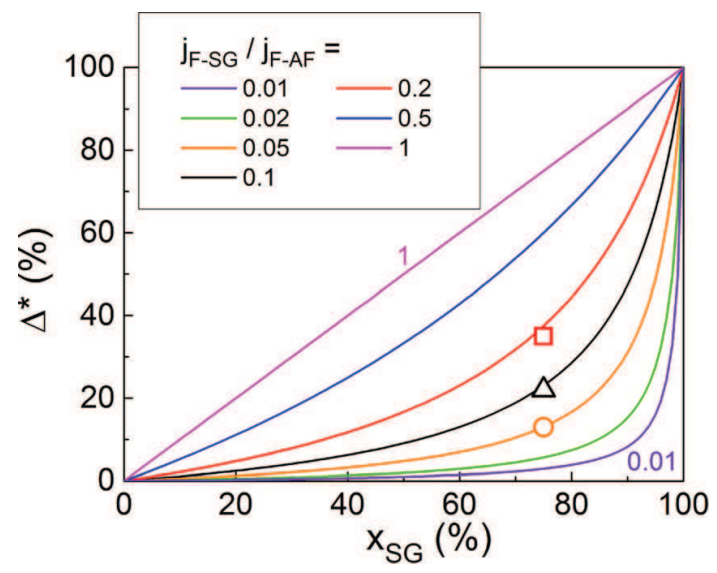

FIG. 8. Calculated variation in the surface fraction $\Delta^{*}$ of the lowtemperature peak of $d H_{\mathrm{E}} / d T_{\mathrm{a}}$ with proportion of SG at F/AF interface, $x_{\mathrm{SG}}$, for various $j_{\mathrm{F}-\mathrm{SG}} / j_{\mathrm{F}-\mathrm{AF}}$ ratios. The open symbols represent values obtained from Monte Carlo simulations.

coupling intensity between small grains (SG) taking into account less stable phases at a ferromagnetic (F)/antiferromagnetic (AF) interface and the $\mathrm{F}$ or $\mathrm{AF}$ grains, which explain experimental results indicating a bimodal blocking temperature distribution. In addition, in these conditions, the coercive field is not dependent on the annealing temperature as for experimental data. Importantly, at $0 \mathrm{~K}$ these two couplings must be approximately smaller or equal to the anisotropy energy of the $\mathrm{SG}\left(K_{S G}^{0} V_{S G}\right)$. The surface coverage of disordered magnetic phases at the interface can, on principle, be extracted from experimental measurements using the protocol presented by Soeya et al., ${ }^{5}$ but our model demonstrated that this calculation requires the ratio between F-SG and FAF couplings to be known. Alternatively, this ratio can be extracted from experimental data, on the condition that it is possible to vary only the surface coverage of the SG without affecting other parameters.

\section{ACKNOWLEDGMENTS}

Simulations were performed at the Centre Régional Informatique et d'Applications Numériques de Normandie (CRIANN) as Project No. 2010006.

\section{APPENDIX A: RELATIONSHIP BETWEEN THE DERIVATIVE $\mathrm{dH}_{\mathrm{E}} / \mathrm{dT}_{\mathrm{a}}$ AND THE BLOCKING TEMPERATURE DISTRIBUTION}

Let us consider the F/AF bilayer in Fig. 2 with a fraction $x_{\mathrm{SG}}$ of SG at the interface. Assuming that a fraction $r$ of AF grains in contact with the F layer and a fraction $r^{\prime}$ of SG are negatively repolarized, the average interfacial coupling per unit surface is

$$
\begin{aligned}
j_{\text {int }}= & {\left[-r\left(1-x_{\mathrm{SG}}\right)+(1-r)\left(1-x_{\mathrm{SG}}\right)\right] j_{\mathrm{F}-\mathrm{AF}} } \\
& +\left[-r^{\prime} x_{\mathrm{SG}}+\left(1-r^{\prime}\right) x_{\mathrm{SG}}\right] j_{\mathrm{F}-\mathrm{SG}} \\
= & (1-2 r)\left(1-x_{\mathrm{SG}}\right) j_{\mathrm{F}-\mathrm{AF}}+\left(1-2 r^{\prime}\right) x_{\mathrm{SG}} j_{\mathrm{F}-\mathrm{SG}} .
\end{aligned}
$$

If the $\mathrm{F}$ layer reverses by uniform rotation, the exchange field $H_{\mathrm{E}}$ at $0 \mathrm{~K}$ will be given by $\mu_{0} H_{\mathrm{E}} M_{\mathrm{F}} t_{\mathrm{F}}=(2 r-1)\left(1-x_{\mathrm{SG}}\right) j_{\mathrm{F}-\mathrm{AF}}+\left(2 r^{\prime}-1\right) x_{\mathrm{SG}} j_{\mathrm{F}-\mathrm{SG}}$

where $M_{\mathrm{F}}$ is the magnetization of each $\mathrm{F}$ grain and $t_{\mathrm{F}}$ is the thickness of the F layer.

The derivative of the exchange field therefore becomes

$$
\mu_{0} M_{F} t_{F} \frac{d H_{E}}{d T_{a}}=2\left[\left(1-x_{S G}\right) j_{F-A F} \frac{d r}{d T_{a}}+x_{S G} j_{F-S G} \frac{d r^{\prime}}{d T_{a}}\right] \text {. }
$$

The fractions $r$ and $r^{\prime}$ are related to the normalized blocking temperature distributions for SG and AF grains in contact with the F layer

$$
r=\int_{0}^{T_{a}} P_{A F}\left(T_{B}\right) d T_{B}, \quad r^{\prime}=\int_{0}^{T_{a}} P_{S G}\left(T_{B}\right) d T_{B},
$$

with $\int_{0}^{+\infty} P_{S G}(T) d T=\int_{0}^{+\infty} P_{A F}(T) d T=1$. Consequently, $\frac{d r\left(T_{a}\right)}{d T_{a}}=P_{A F}\left(T_{a}\right)$ and $\frac{d r^{\prime}\left(T_{a}\right)}{d T_{a}}=P_{S G}\left(T_{a}\right)$.

Equation (A2) can therefore be rewritten as

$$
\begin{aligned}
\frac{d H_{E}\left(T_{a}\right)}{d T_{a}}= & \frac{2}{\mu_{0} M_{F} t_{F}}\left[\left(1-x_{S G}\right) j_{F-A F} P_{A F}\left(T_{a}\right)\right. \\
& \left.+x_{S G} j_{F-S G} P_{S G}\left(T_{a}\right)\right],
\end{aligned}
$$

and the derivative is proportional to the blocking temperature distribution $=\left(1-x_{\mathrm{SG}}\right) P_{\mathrm{AF}}\left(T_{B}\right)+x_{\mathrm{SG}} P_{\mathrm{SG}}\left(T_{B}\right)$ only if $j_{\mathrm{F}-\mathrm{AF}}$ $=j_{\mathrm{F}-\mathrm{SG}}$.

\section{APPENDIX B: EXPRESSION TO DETERMINE THE SURFACE FRACTION OF THE LOW-TEMPERATURE PEAK}

The surface fraction, $\Delta^{*}$, of the low-temperature peak of $d H_{\mathrm{E}} / d T_{\mathrm{a}}$ is equal to $S_{\mathrm{L}} /\left(S_{\mathrm{H}}+S_{\mathrm{L}}\right)$ where $S_{L}=H_{E}\left(T_{\mathrm{a}}=200 \mathrm{~K}\right)$ $-H_{E}\left(T_{\mathrm{a}}=4 \mathrm{~K}\right)$ and $S_{H}+S_{L}=H_{E}\left(T_{\mathrm{a}}=650 \mathrm{~K}\right)-H_{E}\left(T_{\mathrm{a}}=4 \mathrm{~K}\right)$. Assuming uniform rotation of the $\mathrm{F}$ layer, $H_{E}\left(T_{\mathrm{a}}=200 \mathrm{~K}\right)$ can be obtained from Equation (A1) in Appendix A, with $r=0$ and $r^{\prime}=1$ (all SG are negatively repolarized, but the AF grains in contact with the $\mathrm{F}$ layer are not), $H_{E}\left(T_{\mathrm{a}}=4 \mathrm{~K}\right)$ can be obtained with $r=r^{\prime}=0$ since all the entities in contact with the F layer will be positively oriented, while $H_{E}\left(T_{\mathrm{a}}=650 \mathrm{~K}\right)$ can be obtained with $r=r^{\prime}=1$ since all the entities in contact with the $\mathrm{F}$ layer will be negatively repolarized (this gives $\left.H_{E}\left(T_{\mathrm{a}}=650 \mathrm{~K}\right)=-H_{E}\left(T_{\mathrm{a}}=4 \mathrm{~K}\right)\right)$. In this case, $\Delta^{*}$ is given by

$$
\Delta^{*}=\frac{x_{S G} j_{F-S G} / j_{F-A F}}{1-x_{S G}+x_{S G} j_{F-S G} / j_{F-A F}} .
$$

${ }^{1}$ J. Nogués and I. K. Schuller, J. Magn. Magn. Mater. 192, 203 (1999).

${ }^{2}$ A. E. Berkowitz and K. Takano, J. Magn. Magn. Mater. 200, 552 (1999).

${ }^{3}$ K. O'Grady, L. E. Fernández-Outón, and G. Vallejo-Fernandez, J. Magn. Magn. Mater. 322, 883 (2010).

${ }^{4}$ V. Baltz, J. Sort, B. Rodmacq, B. Dieny, and S. Landis, Phys. Rev. B 72, 104419 (2005).

${ }^{5}$ S. Soeya, T. Imagawa, K. Mitsuoka, and S. Narishige, J. Appl. Phys. 76, 5356 (1994).

${ }^{6}$ E. Fulcomer and S. H. Charap, J. Appl. Phys. 43, 4190 (1972).

${ }^{7}$ A. P. Malozemoff, Phys. Rev. B 35, 3679 (1987). 
${ }^{8}$ V. Baltz, B. Rodmacq, A. Zarefy, L. Lechevallier, and B. Dieny, Phys. Rev. B 81, 052404 (2010).

${ }^{9}$ H. Ohldag, A. Scholl, F. Nolting, E. Arenholz, S. Maat, A. Young, M. J. Carey, and J. Stöhr, Phys. Rev. Lett. 91, 017203 (2003).

${ }^{10}$ K. Takano, R. H. Kodama, A. E. Berkowitz, W. Cao, and G. Thomas, J. Appl. Phys. 83, 6888 (1998).

${ }^{11}$ J. Ventura, J. P. Araujo, J. B. Sousa, A. Veloso, and P. P. Freitas, J. Appl. Phys. 101, 113901 (2007).

${ }^{12}$ M. P. Proenca, J. Ventura, C. T. Sousa, M. Vazquez, and J. P. Araujo, Phys. Rev. B 87, 134404 (2013).

${ }^{13}$ F. Spizzo, E. Bonfiglioli, M. Tamisari, A. Gerardino, G. Barucca, A. Notargiacomo, F. Chinni, and L. Del Bianco, Phys. Rev. B 91, 064410 (2015).

${ }^{14}$ K. Akmaldinov, L. Frangou, C. Ducruet, C. Portemont, J. Pereira, I. Joumard, B. Dieny, J. Alvarez-Hérault, and V. Baltz, IEEE Magn. Lett. 6, 3000404 (2015).

${ }^{15}$ V. Baltz, Appl. Phys. Lett. 102, 062410 (2013).

${ }^{16} \mathrm{G}$. Lhoutellier, D. Ledue, R. Patte, F. Barbe, B. Dieny, and V. Baltz, J. Phys. D: Appl. Phys. 48, 115001 (2015).

${ }^{17}$ R. Quey, P. R. Dawson, and F. Barbe, Comput. Methods Appl. Mech. Eng. 200, 1729 (2011).
${ }^{18}$ D. Choo, R. W. Chantrell, R. Lamberton, A. Johnston, and K. O'Grady, J. Appl. Phys. 101, 09E521 (2007).

${ }^{19}$ B. Craig, R. Lamberton, A. Johnston, U. Nowak, R. W. Chantrell, and K. O'Grady, J. Appl. Phys. 103, 07C102 (2008).

${ }^{20}$ M. D. Stiles and R. D. McMichael, Phys. Rev. B 60, 12950 (1999).

${ }^{21}$ D. W. Heermann, Computer Simulation Methods in Theoretical Physics, 2nd ed. (Springer, Berlin, 1990).

${ }^{22}$ K. Binder and D. W. Heermann, Monte Carlo Simulations in Statistical Physics, 2nd ed. (Springer, Berlin, 1990).

${ }^{23}$ D. Ledue, A. Maitre, F. Barbe, and L. Lechevallier, J. Magn. Magn. Mater. 372, 134 (2014).

${ }^{24}$ D. Brinis, A. Laggoun, D. Ledue, and R. Patte, J. Appl. Phys. 115, 173906 (2014).

${ }^{25}$ L. Lechevallier, A. Zarefy, R. Lardé, H. Chiron, J.-M. Le Breton, V. Baltz, B. Rodmacq, and B. Dieny, Phys. Rev. B 79, 174434 (2009).

${ }^{26}$ L. Lechevallier, A. Zarefy, F. Letellier, R. Lardé, D. Blavette, J.-M. Le Breton, V. Baltz, B. Rodmacq, and B. Dieny, J. Appl. Phys. 112, 043904 (2012).

${ }^{27}$ G. Vallejo-Fernandez, L. E. Fernandez-Outon, and K. O'Grady, Appl. Phys. Lett. 91, 212503 (2007). 